\title{
Erratum to: Autonomic symptoms in idiopathic REM behavior disorder: a multicentre case-control study
}

\author{
Luigi Ferini-Strambi $\cdot$ Wolfgang Oertel $\cdot$ Yves Dauvilliers $\cdot$ Ronald B. Postuma $\cdot$ Sara Marelli \\ Alex Iranzo • Isabelle Arnulf • Birgit Högl • Raffaele Manni · Tomoyuki Miyamoto • \\ Maria-Livia Fantini · Monica Puligheddu • Poul Jennum • Karel Sonka · Joan Santamaria • \\ Marco Zucconi · Paola M. V. Rancoita $\cdot$ Smeranda Leu-Semenescu • Birgit Frauscher • \\ Michele Terzaghi · Masayuki Miyamoto • Marcus Unger · Karin Stiasny-Kolster • Alex Desautels • \\ Christina Wolfson $\cdot$ Amélie Pelletier · Jacques Montplaisir
}

Published online: 14 December 2014

(C) Springer-Verlag Berlin Heidelberg 2014

\section{Erratum to: J Neurol (2014) 261:1112-1118}

DOI 10.1007/s00415-014-7317-8

The given name and family name of the following author Birgit Högl are represented incorrectly in the original version of the article. The correct author name should be: Birgit Högl.
The online version of the original article can be found under doi:10.1007/s00415-014-7317-8.

\section{Ferini-Strambi $(\bowtie) \cdot$ S. Marelli · M. Zucconi}

Department of Clinical Neuroscience, Sleep Disorders Center,

Università Vita-Salute San Raffaele, Via Stamira d' Ancona 20,

20127 Milan, Italy

e-mail: ferinistrambi.luigi@hsr.it

W. Oertel · K. Stiasny-Kolster

Department of Neurology, Philipps-University, Marburg,

Marburg, Germany

\section{Y. Dauvilliers}

Neurological Department, University Hospital, Montpellier, France

R. B. Postuma - A. Desautels - A. Pelletier · J. Montplaisir Centre d'Études Avancées en Médecine du Sommeil, Hôpital du Sacré-Cœur de Montréal, Montreal, Canada

\section{A. Iranzo $\cdot$ J. Santamaria}

Neurology Service, Hospital clinic de Barcelona, Barcelona, Spain

I. Arnulf $\cdot$ S. Leu-Semenescu

Unité des pathologies du sommeil, Hôpital Pitié-Salpêtrière, APHP and Inserm U975-CRICM-Pierre and Marie Curie University, Paris Cedex 13, France
B. Högl · B. Frauscher

Department of Neurology, Innsbruck Medical University, Innsbruck, Austria

R. Manni · M. Terzaghi

Sleep and Epilepsy Unit, IRCCS C. Mondino National Institute of Neurology Foundation, Pavia, Italy

T. Miyamoto $\cdot$ M. Miyamoto

Department of Neurology, Dokkyo Medical University

Koshigaya Hospital, Koshigaya, Saitama, Japan

M.-L. Fantini

Department of Neurosciences, Sleep Disorders Center,

University of Turin, Ospedale San Giovanni Battista-Molinette,

Turin, Italy

M. Puligheddu

Department of Cardiovascular and Neurological Sciences, Sleep

Center, University of Cagliari, Cagliari, CA, Italy

P. Jennum

Danish Center for Sleep Medicine, University of Copenhagen,

Copenhagen, Denmark 


\section{K. Sonka}

Department of Neurology and Center of Clinical Neuroscience, First Faculty of Medicine and General University Hospital in

Prague, Charles University in Prague, Prague, Czech Republic

P. M. V. Rancoita

CUSSB (University Centre for Statistics in the Biomedical Sciences), Vita-Salute San Raffaele University, Milan, Italy

M. Unger

Department of Neurology, Saarland University Homburg/Saar, Saar, Germany

\section{Wolfson}

Department of Epidemiology, Biostatistics and Occupational Health, McGill University, Montreal, Canada 